\title{
The SARS-CoV-2 Outbreak: an Epidemiological and Clinical Perspective
}

\author{
Rebecca S. Y. Wong ${ }^{1}$ (D) \\ Accepted: 23 September 2020 / Published online: 29 September 2020 \\ (C) Springer Nature Switzerland AG 2020
}

\begin{abstract}
The severe acute respiratory syndrome coronavirus 2 (SARS-CoV-2) outbreak started with the detection of an increasing number of pneumonia cases of unknown origin in Wuhan, China, since December 2019. The disease caused by SAS-CoV-2 was subsequently named coronavirus disease 2019 (COVID-19). Currently, the ongoing COVID-19 pandemic poses a global health concern with more than 28.9 million confirmed cases, taking away the lives of more than 900,000 people worldwide. To prevent further spread of the disease, an understanding of the clinical characteristics and how the disease spread is essential, especially for an emerging disease like COVID-19. Individuals who are infected with SARS-CoV-2 show diverse clinical features, and the disease severity can range from asymptomatic to death. The disease has been shown to affect not just the respiratory system but also other systems of the body. This review will discuss the pulmonary and extra-pulmonary clinical manifestations of COVID19 in general, as well as the clinical characteristics in different groups of patients such as children, the elderly, pregnant women, patients with comorbidities and those with a compromised immunity. It will also critically examine existing evidence from relevant studies and discuss the SARS-CoV-2 outbreak from an epidemiological perspective. With the easing of control measures in many countries after months of lockdown, it is important to revisit the lessons learnt from research, as the world enters a new normal with the coexistence of SARS-CoV-2.
\end{abstract}

Keywords SARS-CoV-2 $\cdot$ COVID-19 $\cdot$ Outbreak $\cdot$ Pandemic $\cdot$ Epidemiological characteristics $\cdot$ Clinical characteristics

\section{Introduction}

In public health, battling life-threatening emerging diseases is always challenging as much has yet to be learnt and discovered about these diseases. The recent ongoing coronavirus $(\mathrm{CoV})$ outbreak is caused by a novel $\mathrm{CoV}$ initially named 2019-nCoV, first identified in Wuhan, Hubei Province, China, with increasing number of pneumonia cases being detected in December 2019. The problem has escalated and drawn international attention due to the rapid spread of the disease in China and other parts of the world. As a result of the increasing magnitude of the global health threat, the World Health Organisation (WHO) has declared the 2019-nCoV as a

This article is part of the Topical Collection on Covid-19

Rebecca S. Y. Wong

rebecca@segi.edu.my; rebeccawongsy@gmail.com

1 Faculty of Medicine, SEGi University, No. 9, Jalan Teknologi, Taman Sains Selangor, Kota Damansara, PJU 5, 47810 Petaling Jaya, Selangor, Malaysia global health emergency on 31 January 2020 [1]. The virus was later renamed as severe acute respiratory syndrome coronavirus 2 (SARS-CoV-2) by the International Committee on Taxonomy of Viruses (ICTV) and the WHO announced that the disease caused by the virus is to be called coronavirus disease 2019 (COVID-19) on 11 February 2020 [2]. The outbreak has alarmed scientists and healthcare professionals, government authorities and the World Health Organisation, with the burning needs to come up with plans and policies for diagnosis, case management, surveillance and risk management, as well as infection prevention and control.

In response to the rapidly growing number of confirmed cases and deaths, some measures taken by the Chinese authorities include the quarantine of millions of its citizens with the unprecedented lockdown of many cities, in an attempt to contain the virus and slow down the spread of the disease [3]. On the other hand, as early as end of January or early February 2020, some countries like Australia and the USA have imposed travel bans on China, whereas some other countries have cancelled flights from China, which plunge the latter into deepening isolation as a result of the outbreak. Major 
companies like Google and Facebook have banned travel to China while Apple and Starbucks have shut their stores in the country [4]. The lockdown and travel bans have a huge impact on the global economy as such a rare, abrupt and open-ended freeze out involving a vital economic centre like China is likely to lead to detrimental economic effects that ripple across the world.

On 11 March 2020, WHO declared the COVID-19 outbreak a pandemic [5]. As of 13 September 2020, there have been over 28.9 million confirmed cases and more than 900,000 deaths reported since the SARSCoV-2 outbreak [6]. The number of publications on various aspects of the SARS-CoV-2 outbreak is on the rise since early 2020. New information and discoveries are being added to the published literature as there is a growing body of research in an attempt to unveil the mysteries of the novel coronavirus and to better understand the new pathogen. This review aims to discuss the clinical and epidemiological aspects as well as the transmission dynamics of the SARS-CoV-2 outbreak. Thus far, the clinical features of COVID-19 have been described by many. However, the current review will examine the clinical features observed in different categories of patients such as children, the elderly, pregnant women, the immunocompromised as well as patient with comorbidities to illustrate the diversity of the clinical presentation of COVID-19.

\section{Epidemiological Parameters}

Success in the control of an outbreak often lies in the understanding of how the disease gets from one individual to another. Interesting and valuable information can be deduced or inferred from several epidemiological parameters. This is important from a public health perspective, as knowledge on these epidemiological parameters of the disease helps provide insights concerning the appropriate control measures to be used in order to contain the disease and prevent further spread. This section will discuss the various parameters that are related to the transmission of a contagious communicable disease such as COVID-19.

\section{Incubation Period}

In a study analysing the first 425 confirmed cases in Wuhan by 22 January $2020, \mathrm{Li}$ et al. reported that prior to January $2020,55 \%$ of the cases were linked to the Huanan Seafood Wholesale Market in Wuhan, whereas such linkage was observed in only $8.6 \%$ of subsequent cases. Among these cases, there was a mean incubation period of 5.2 days (95\% confidence interval [CI], 4.1 to 7.0 ), with the 95 th percentile of the distribution at 12.5 days. The epidemic was shown to double in size every 7.4 days in its early stages. Findings of the study indicate the evidence of human-to-human transmission among close contacts since mid-December 2019. Due to its earlier link to the Huanan Seafood Wholesale Market in Wuhan and high genomic similarities between SARS-CoV-2 and bat coronaviruses, it has been suggested that COVID-19 is a disease of zoonotic origin with animals such as bats as the reservoir of SARS-CoV-2 [7].

In another study, Chen et al. investigated 99 patients consisting of 67 men and 32 women with 2019-nCoV pneumonia in Wuhan Jinyintan Hospital from 1 January to 20 January 2020. It was shown that $49 \%(n=49)$ of the patients had an exposure history to the Huanan Seafood Market. The average age of these patients was 55.5 years (SD 13.1 years) [8]. Laeuer et al. analysed 181 cases occurring before 24 February 2020 outside the province of Hubei, in which 108 were from outside China. Most of these cases either had a travel history to Wuhan or had contact with travellers from the region. An estimated median incubation period of 5.1 days (95\% CI, 4.5-5.8 days) was reported. By 11.5 days (95\% CI, 8.2-15.6 days), $97.5 \%$ among those infected had showed symptoms of infection. The study also reported that to capture $>99 \%$ of symptomatic cases, a monitoring duration of $>$ 14 days was required [9].

\section{Serial Interval}

The serial interval (also known as generation time, $T_{\mathrm{g}}$ ) refers to the average duration taken from the time an individual being infected to the time he/she infects others. An empirical estimate of $T_{\mathrm{g}}$ can be obtained by observing the time from illness onset of a primary case (infector) to the secondary case's illness onset and is important in our understanding of case generation and disease transmissibility [10]. Linking dates of onset for the infector-infectee pairs is crucial to estimates of $T_{\mathrm{g}}$. However, these links may not be easily established.

Using contact tracing data from cases reported early in the outbreak in Hubei Province, an epidemiology study reported a mean serial interval of 7.5 days [7]. Another study reported an estimated mean serial interval at 4.0 days $(95 \%$ credible interval $3.1,4.9)$ for 28 infectorinfectee pairs (both certain and probable pairs). Among the dataset, a subset of 18 pairs (certain pairs) yielded an estimated median serial interval at 4.6 days (95\% credible interval 3.5, 5.9). The close-to or shorter-than-median incubation period serial interval suggests pre-symptomatic transmissions for a considerable proportion of secondary transmissions [11]. On the other hand, Liu et al. estimated the mean serial interval to be 5.81 days $( \pm 3.24)$ using a total of 116 pairs of infector-infectee that met strict inclusion criteria [12]. 


\section{Basic and Effective Reproductive Numbers}

Other than the incubation period and serial interval, researchers are interested in the basic reproductive number $\left(R_{0}, R\right.$ naught or $R$ zero) and effective reproductive number $\left(R_{e}\right.$ or $\left.R_{t}\right)$. In order to better understand the transmissibility of SARS-CoV-2, an understanding of the definition and indication of $R_{0}$ is crucial. $R_{0}$ can be defined as the average number of people who can be infected by an infectious person in a completely naïve population. If $R_{0}$ is $>1$, it means that the infection is likely to spread exponentially. On the contrary, if $R_{0}$ is $<1$, it means that the infection is spreading slowly and will eventually die off. For example, if the $R_{0}$ is 2 , it means one infected person has the potential of spreading the disease to two other persons [13].

In general, $R_{0}$ is affected by population density and the initial proportion of susceptible people, the organism's infectiousness, as well as the case disappearance rate (either by recovery or death). It is important to note that $R_{0}$ is not a rate and, therefore, has no units of time such as in doubling time. On the other hand, $R_{e}$ or $R_{t}$, refers to the average number of secondary cases per infectious case in a population of susceptible and non-susceptible hosts [13]. Therefore, $R_{t}$ is usually lower than $R_{0}$ because as the previously infected people become immune to the disease, the number of people who can be infected and become secondary cases decreases. If $R_{t}$ is $>1$, the number of cases will increase. If it is $<1$, the number of cases will decrease. If $R_{t}=1$, the disease is considered endemic.

Liu et al. explored $R_{0}$ of COVID-19 by reviewing 12 related studies. $R_{0}$ was estimated be 1.4-6.49 (mean $=3.28$, median $=2.79$, interquartile range $=1.16$ ), which was higher than the WHO estimate of 1.4-2.5 $($ mean $=1.95)$. However, it is noteworthy that estimates of $R_{0}$ depend on the model used. It was observed that the two studies that used stochastic methods gave a $R_{0}$ estimate of $2.2-2.68($ mean $=2.44)$. Six studies that used mathematical methods and three studies that used statistical methods gave estimates of $1.5-6.49($ mean $=4.22)$ and $2.2-3.58$ (mean $=2.67)$ respectively [14].

Using data retrieved from John Hopkins University's COVID-19 data repository, Caicedo-Ochoa et al. estimated the $R_{t}$ of SARS-CoV-2 infection in seven Latin American countries (i.e. Brazil, Chile, Colombia, Ecuador, Mexico, Panama and Peru) during the first 10 days of the outbreak, during which the incidence was highest. They also compared the $R_{t}$ values with that of Spain and Italy for the same interval. The $R_{t}$ values in decreasing order were $3.95(3.70-4.21)$ for Ecuador, 2.67 (3.25-4.13) for Panama, 2.91 (2.60-3.23) for Brazil, 2.67 (2.45-2.89) for Chile, 2.67 (2.3802.98) for Colombia, 2.36 (2.11-2.63) for Peru and 2.42 (2.14-2.72) for Mexico whereas the $R_{t}$ values for Spain and Italy were $2.90(2.67-3.14)$ and $2.83(2.70-2.96)$ respectively. The study therefore concluded that the seven Latin American countries had a high $R_{t}$ during the initial stages of the COVID-19 outbreak [15].

\section{Doubling Time}

As its name implies, the doubling time of a disease in a given period refers to the time required for the number of cases to double. A short doubling time means rapid increase in the number of cases and vice versa. Therefore, the doubling time gives us useful information concerning the impact of interventions during an outbreak. Many factors play a role in the doubling time of an infectious disease, e.g. $R_{0}, R_{t}$, incubation period, latent period and serial interval. Even if two diseases have to same $R_{0}$, the doubling time may differ due to a different serial interval. On the other, doubling time for a disease may differ from one place to another due to a different $R_{0}$ or $R_{t}$.

An earlier study (from 20 January to 9 February 2020) in China reported that doubling times of the COVID-19 epidemic for Hunan Province, Hubei Province and Xinjiang Province were 1.4 days (95\% CI 1.2-2.0), 2.5 days (95\% CI 2.4-2.6) and 3.1 days (95\% CI 2.1-4.8) respectively [16]. On the other hand, analysis of data obtained from several European countries in February and March 2020 indicates that the initial doubling times of COVID-19 was approximately 3 days or less using semiparametric and generalised linear methods, until social distancing measures were in place. The study also reported that the increase in the number of cases was slowed by these measures, and the effects were typically observed only in 9 days (i.e. 3 doubling times) after their implementations. The study concluded that the estimation of doubling time, which reflects that the temporal patterns are more crucial than the estimation of $R_{0}$ for initiation of interventions, especially for low- and middle-income countries that are in their early stages of the outbreak [17].

\section{Modes of Transmission}

An infectious agent can be transmitted from its natural reservoir to hosts via different routes. Many studies have looked into the modes of transmission of SARS-CoV-2 infection and both direct and indirect modes have been reported in the published literature.

\section{Human-to-Human Transmission}

Currently, human-to-human transmission via respiratory droplets is the primary route through which the infection spread aggressively in different parts of the world. Humanto-human transmission can take place in one of the three ways: (1) asymptomatic transmission, (2) pre-symptomatic transmission and (3) symptomatic transmission. Of particular 
concern is asymptomatic transmission as carriers of SARSCoV-2 without symptoms may not be identified unless they are tested positive by reverse-transcription polymerase chain reaction (RT-PCR) or other laboratory tests.

One study in China reported a young 22-year-old male who spread SARS-CoV-2 infection to his contacts (1 relative and 6 classmates, all of which were youngsters from 16 to 23 years) just after a few-hour contact during the incubation period, when he was totally asymptomatic [18], suggesting that the disease is highly infectious during the incubation period. Another study reported a 20-year-old presumed asymptomatic carrier of SARS-CoV-2, who was believed to have spread the infection to 5 relatives. At the time of contact with the 5 relatives, the carrier had no symptoms and her subsequent chest computer tomography (CT) and lymphocyte count were normal. However, her 5 relatives were tested positive for COVID-19 later and were all symptomatic with abnormalities on chest CT [19].

Researchers have attempted to estimate the asymptomatic portion of the COVID-19. One study estimated asymptomatic infections on the Diamond Princess cruise ship hosting 3711 people. After a former passenger was tested positive for COVID-19, other passengers on the ship underwent quarantine for 2 weeks. A total of 634 passengers were subsequently tested positive, with 306 symptomatic and 328 asymptomatic cases. Using statistical modelling, asymptomatic infections was estimated to be $17.9 \%$ (95\% credible interval $15.5-$ 20.2\%) [20]. Another study conducted on Japanese nationals evacuated from Wuhan, China, on chartered flights gave an estimated asymptomatic ratio of $30.8 \%$ (95\% CI, 7.7-53.8\%) among evacuees [21].

\section{Vertical and Transplacental Transmission}

A systemic review conducted on 70 newborns (in nine independent studies) born to mothers with confirmed COVID-19 infection revealed the possibility of vertical transmission. Out of the 70 newborns, $92.9 \%(n=65)$ were tested negative within the first few hours or days after birth. However, vertical transmission could not be excluded, as the oropharyngeal or nasopharyngeal swabs of $5.7 \%(n=4)$ of the newborns were tested positive within days of birth. The throat swab of one newborn was tested negative. However, IgM and IgG testing was positive in this case, suggesting possible in utero infection and transplacental transmission. Therefore, transplacental or vertical transmission could not be ruled out in $5(7.1 \%)$ of out the 70 newborns [22].

\section{Fomite Transmission}

Other than aerosol transmission via sneezing, coughing, etc., research has implied that indirect routes such as contacting surfaces contaminated with SARS-CoV-2 are plausible routes of transmission. One study demonstrated that SARS-CoV-2 was able to remain viable in aerosols for $3 \mathrm{~h}$, and that viable virus could be detected on plastic and stainless steel for up to $72 \mathrm{~h}$. On the other hand, viable virus was less stable on surfaces like copper (not detectable after $24 \mathrm{~h}$ ) and cardboard (not detectable beyond $8 \mathrm{~h}$ ) [23]. Therefore, both aerosol and fomite transmissions play a role in the rapid spread of COVID19.

In another study, Chin et al. reported that SARS-CoV-2 is highly stable and resistant for a long time at $4{ }^{\circ} \mathrm{C}$ whereas at $70^{\circ} \mathrm{C}$, the time required to inactivate the virus was decreased to $5 \mathrm{~min}$. At room temperature, the virus was also found to be extremely stable in $\mathrm{pH}$ ranges of 3-10. Adding various disinfectants at working concentrations to SARS-CoV-2 cultures at room temperature revealed a non-detection of virus after an incubation period of $5 \mathrm{~min}$. The virus was inoculated on various objects in the same study. No virus was detected on printing and tissue papers after $3 \mathrm{~h}$ of incubation, while the virus was not found on treated wood and cloth on day 2. It was not detected on glass and banknotes on day 4 and stainless steel and plastic, on day 7. However, it was found on a surgical mask's outer layer on day 7 [24].

\section{Environmental Factors Affecting Transmission}

Studies have shown that various environmental factors may affect transmission of COVID-19. Scientists are interested in the relationship between various environmental factors and the transmission of SARS-CoV-2 infection. It was initially believed that with rising temperatures, the spread of the disease will slow down and the number of cases will reduce. While some studies showed that environmental factors such as temperature and humidity are associated with the transmission rates of COVID-19, there is no strong evidence to suggest that the disease will eventually become dormant as summer approaches thus far. Studies on the relationship between environmental factors and COVID-19 transmission are often observational studies with various confounding factors such as population statistics and public health preventive measures (e.g. lockdowns or movement restrictions, etc.). Meteorological data are often obtained through regional data extrapolation while ascertainment methods of cases may vary for different regions.

Bhattacharjee investigated the relationship between three environmental factors: (1) maximum relative humidity (RHmax), (2) maximum temperature (Tmax) and (3) highest wind speed (WSmax) and the daily number of confirmed COVID-19 cases in four cities in China (i.e. Beijing, Chongqing, Shanghai and Wuhan) and five cities in Italy (i.e. Bergamo, Brecia, Cremona, Lodi and Milano). It was reported that the relationship between daily number of cases 
and RHmax and WSmax was mostly negligible, whereas the relationship with Tmax ranged from negligible to moderate [25]. On the other hand, Luo et al. collected epidemiological data from Hong Kong, Japan, South Korea, Singapore, Taiwan, Thailand and different regions of China to investigate the relationship between (1) absolute humidity and (2) temperature and local exponential growth of the outbreak. Absolute humidity was positively correlated to case increase while weather temperature was weakly and negatively corrected to case increase [26].

Meteorological and epidemiological data collected from 166 countries (other than China), suggested that daily new cases and deaths were related to relative humidity and temperature, after controlling for confounding factors such as national population median age, population density and wind seed. Both relative humidity and temperature exhibited a negative relationship with the daily new cases and deaths. With every $1{ }^{\circ} \mathrm{C}$ rise in temperature, there was a $3.08 \%(95 \%$ CI $1.53 \%$, $4.63 \%)$ and $1.19 \%$ (95\% CI $0.44 \%, 1.95 \%)$ decrease in daily new cases and new deaths respectively. As for every $1 \%$ increase in relative humidity, a $0.85 \%(95 \%$ CI $0.51 \%, 1.19 \%)$ and $0.51 \%(95 \%$ CI $0.34 \%, 0.67 \%)$ decrease in daily new cases and new deaths were observed respectively [27].

\section{Clinical Characteristics in General}

SARS-CoV-2 infection mainly affects the respiratory system, although involvement of other organs/systems is not uncommon. The clinical presentation of COVID-19 is diverse, ranging from asymptomatic to very severe illness, which can lead to death. To understand the clinical aspects of COVID-19, it is necessary to have an understanding of the pathogenesis of the disease. Once a person is infected with SARS-CoV-2, the inhaled virus binds to ACE2 receptor to gain entry into host cells in the respiratory tract [28]. During this initial stage (first 1 or 2 days) of the infection, the patient is asymptomatic, but the virus may be detected in the nasal swabs. As the virus propagates locally, individuals in this stage are infectious despite the low viral load with a limited immune response in the body. In the next few days, the virus continues to propagate down the conducting airways, triggering a more vigorous immune response [29]. During this time, the patient begins to manifest the common symptoms of COVID-19, such as fever, sore throat and dry cough.

In approximately $80 \%$ of the cases, the disease is mild to moderate, confining to the upper respiratory tract. However, $20 \%$ of the cases progress to more severe disease with pulmonary infiltrates, as the virus reaches the lower respiratory tract [30]. It is this category of patients who present with dyspnoea and may progress to acute respiratory distress syndrome (ARDS) as the disease continues to worsen. Some of the underlying immune responses in severe cases include lymphopenia and increased release of pro-inflammatory cytokines resulting in an ARDS-inducing "cytokine storm," which may be followed by multiorgan failure leading to death [31, 32].

Although COVID-19 is mainly a respiratory disease characterised by pulmonary manifestations, reports on extrapulmonary manifestations are not uncommon in the published literature. Some of these include gastrointestinal, cardiac, neurological and cutaneous manifestations, features of renal and liver dysfunction, as well as taste and smell disorders [33, 34]. Patients with gastrointestinal involvement may present with symptoms such as diarrhoea, vomiting, abdominal pain and loss of appetite [35] whereas neurological manifestations such as encephalitis, encephalopathy, stroke and Guillain Barre Syndrome have been reported [9]. Patients with cutaneous presentation may exhibit erythematous pustules or vesicles, maculopapular or vesicular eruptions, urticaria, liverdo and necrosis [36]. On the other hand, acute cardiac injury and raised cardiac troponins are abnormalities observed in some patients with COVID-19, whereas patients with existing cardiovascular disease tend to have a worse outcome compared with those without. The underlying mechanisms of the injury are said to be related to direct injury to cardiomyocytes or indirectly due to overwhelming systemic inflammation [37].

\section{Clinical Features in Children}

In one study, Dong et al. investigated the clinical presentation of 2143 paediatric lab-confirmed $(n=731)$ and suspected cases ( $n=1412)$ of COVID-19 with an interquartile age range from 2 to 13 years. It was found that $>90 \%$ of these patients demonstrated either no symptoms, or mild to moderate symptoms without showing any significant gender difference. The study concluded that children of all ages, especially infants, were susceptible and vulnerable to the infection, even though they presented with milder disease compared with adults [38].

Despite earlier studies demonstrating a milder course of the disease in children, recent studies have reported cases of paediatric patients with severe disease requiring intensive care. In one of these cases, Greene et al. described toxic shock-like syndrome in an 11-year-old girl who was tested COVID-19 positive. During her first visit to the Emergency Department (ED), the patient presented with fever, rash and pharyngitis. However, within $48 \mathrm{~h}$, she returned to the ED with multiorgan injury, systemic inflammation and circulatory shock and was admitted to the paediatrics ICU due to cardiac and renal dysfunction, as well as fluid-refractory hypotension. While receiving supportive therapy in the PICU, the patient was also given steroids and intravenous immunoglobulin (IVIG) for possible incomplete Kawasaki disease. Dramatic improvement was observed $<24 \mathrm{~h}$ and the patient was subsequently discharged home [39]. 
In France, a study on 21 children and adolescents aged from 3.7-16.6 years examined the relation between COVID19 and Kawasaki disease. It was reported that $90 \%(n=19)$ of the patients had evidence of COVID-19 infection with $81 \%$ $(n=17)$ requiring treatment in the ICU. All these children had clinical features of Kawasaki disease with $57 \%(n=12)$ and $76 \%(\mathrm{n}=12)$ presented with Kawasaki disease shock syndrome and myocarditis respectively. All of them were treated with IVIG and $48 \%(n=10)$ of the patients, with corticosteroids, after which all were discharged home. Findings from this study suggest that Kawasaki-like multisystem inflammatory syndrome might be related to SARS-CoV-2 infection [40].

\section{Clinical Features in Pregnant Women}

Studies have investigated the clinical features and outcomes of COVID-19 in pregnant women. Cao et al. reported the clinical analysis of 10 pregnant women in their third trimester till the postpartum period. All 10 women was tested COVID-19 positive and presented with mild disease. During the period of the study, none of the women developed severe disease, severe respiratory distress or needed to use mechanical ventilation. However, lung abnormalities were observed in the chest CT of all patients. Two patients had vaginal delivery, 2 had intrapartum caesarean section and 6 had elective caesarean section A total of 11 newborns (9 singletons and 1 pair of twins) were born during the study period. All the 11 newborns had an Apgar score of 8-9 and 10 at $1 \mathrm{~min}$ and 5 min after birth respectively, and none of them had neonatal asphyxia or presented with fever, cough or diarrhoea. Five of the newborns underwent COVID-19 testing, and none of them were tested positive. The study concluded that COVID-19 is not an indication for caesarean section [41].

On the other hand, a systemic review and metanalysis on 136 women in 24 studies reported that fever (62.9\%), cough $(36.8 \%)$ and sore throat $(22.6 \%)$ were the commonest clinical features in pregnant women with COVID-19 while dyspnoea (15.7\%) and diarrhoea $(15.6 \%)$ were less frequently reported. The common lab findings were increased C-reactive protein levels (57\%) and lymphocytopenia $(50 \%)$ whereas ground-glass opacity was observed in the chest $\mathrm{CT}$ in $81.7 \%$ of the patients. There was a preterm birth rate of $37.7 \%$ and $76 \%$ of women underwent caesarean section. One maternal death, 3 foetal deaths (at gestational ages of 34, 31 and 30 weeks) were reported and 2 newborns were tested COVID-19 positive. It was concluded that the clinical presentation of pregnant women did not vary from the general population, however, compared with international averages there was an increased preterm birth rate and caesarean delivery rates [42].

\section{Immunocompromised Individuals and COVID-19}

Overwhelming inflammatory reactions due to a cytokine storm have been described in patients with severe COVID19 , which can result in organ damage and failure [7, 8]. Therefore, some believe that the anti-inflammatory effects of immunosuppression may be protective and helps to mitigate the cytokine storm associated with poor outcomes. However, findings on the outcomes of immunocompromised individuals with COVID-19 are contradicting with some studies reporting unfavourable outcomes and others suggesting otherwise. For example, one study reported that a higher risk of severe disease and death was not observed in HIV patients with COVID-19 [43] whereas another study reported that HIV was associated with a doubling of mortality risk in COVID19 patients [44].

One study investigated COVID-19 mortality in 800 cancer patients and demonstrated that those who were on various anti-cancer treatments did not have a significant higher risk of mortality. Out of the 800 cancer patients, more than half $(52 \%, n=412)$ had mild disease. Out of the $28 \%(n=226)$ patients who died, a significant association between the risk of death and (1) advancing age (odds ratio 9.42 [95\% CI 6.56-10.02]; $p<0.0001$ ), (2) male gender (1.67 [1.19-2.34]; $p=0.003)$ and (3) comorbidities (e.g. hypertension $(1.95[1.36-2.80] ; p<0.001)$ or cardiovascular disease (2.32 [1.47-3.64]) was observed. When these factors were adjusted, chemotherapy, hormonal therapy, targeted therapy, immunotherapy and radiotherapy use had no significant effect on mortality from COVID-19 when comparing patients who received these therapies versus those who did not [45].

On the contrary, another study conducted on 205 cancer patients with confirmed COVID-19 reported contradicting findings. In the study, $89 \%(n=183)$ of the patients had solid tumours while $11 \%(n=22)$ had haematological malignancies. Out of the 205 patients, $30 \%(n=54)$ had anti-cancer therapies within 4 weeks prior to symptom onset. A total of $40(20 \%)$ patients died during their hospital stay. Patients with haematological malignancies had a poorer prognosis than those with solid tumours whereas a higher percentage of deaths $(41 \%)$ was observed among patients with haematological malignancies compared with those with solid tumours (17\%) (hazard ratio for death 3.28 [95\% CI 1.56-6.91]; log rank $p=0 \cdot 0009$ ). The risk factors of death were chemotherapy within 4 weeks prior to symptom onset (odds ratio [OR] 3.51 [95\% CI 1.16-10.59]; $p=0.026$ ) and male gender (OR 3.86 [95\% CI 1.57-9.50]; $p=0.0033$ ). Therefore, the study concluded that hospitalised cancer patients with COVID-19 had a high case fatality rate while being male and receiving chemotherapy prior to symptom onset were unfavourable prognostic factors among this group of high-risk patients [46]. 


\section{Comorbidities and COVID-19}

Studies have shown that old age and comorbidities are related to the severity of COVID-19 and death. In general, COVID-19 is more lethal among the elderly. This can be illustrated by comparing the case fatality rates of countries with a high proportion of elderly in their population. For example, 23\% of Italy's population consisting of people $\geq 65$ years in 2019 , and the country was hard-hit by the outbreak. Deaths in the country were mainly among elderly males with multiple comorbidities. It was reported that Italy had a considerable higher overall case fatality rate (CFR) than that of China $(7.2 \%$ vs $2.3 \%)$. For the 0 to 69 years age groups, the CFR is similar for both countries. However, the CFR in individuals $\geq 80$ years was shown to be higher in Italy. The higher overall CFR in Italy can be partly explained by a difference in the case distribution in the two countries; in Italy $37.6 \%$ of cases were $\geq 70$ years, whereas in China only $11.9 \%$ of the cases were $\geq 70 \%$ [47].

On the other hand, a study in the USA consisting of 5700 patients admitted to 12 hospitals reported the commonest comorbidities being hypertension $(56.6 \%$, $n=3026)$, obesity $(41.7 \%, n=1737)$ and diabetes mellitus $(33.8 \%, n=1808)$. The median age of these patient was 63 years [interquartile range (IQR), 52-75].The percentage of patients admitted to the intensive care unit was $14.2 \%(n=373)$ with a median age of 68 years (IQR, 56-78). Of those who received mechanical ventilation $(n=320)$, older patients ( $>65$ years) had a higher mortality rate $(97.2 \%)$ than patients in the 18 to 65 -year group. However, there was no death among those < 18 years of age. Among the patients who died (24.5\%, $n=282$ ), diabetic patients were more likely to be treated with invasive mechanical ventilation or given ICU care compared with non-diabetic patients [48].

Some findings of the study are in tandem with those of another study conducted on 1590 hospitalised patients from 575 hospitals in China, who had laboratory-confirmed COVID-19. The commonest comorbidities were hypertension $(16.9 \%, n=269)$, other cardiovascular diseases $(3.7 \%, n=$ 59), cerebrovascular diseases and diabetes mellitus (both $1.9 \%, n=30$ ). With age and smoking status adjusted, the risk factors identified were chronic obstructive pulmonary disease (hazard ratio [HR] 2.681, 95\% CI, 1.424-5.048), diabetes mellitus (HR 1.59, 95\% CI 1.03-2.45), hypertension (HR $1.58,95 \%$ CI 1.07-2.32) and malignancy (HR 3.50, 95\% CI 1.60-7.64) for those reaching the composite end-points (i.e. ICU admission, invasive ventilation or death). The HR in patient with at least one comorbidity and $\geq 2$ comorbidities was 1.79 (95\% CI 1.16-2.77) and 2.59 (95\% CI 1.61-4.17) respectively [49].

\section{Conclusions}

The current COVID-19 pandemic is a global health threat, impacting the lives of people in many aspects worldwide. From this review, it can be deduced that SARS-CoV-2 has a serial interval close-to or shorter than the incubation period. The fact that SARS-CoV-2 is highly stable under various conditions at room temperature and lingers on different surfaces up to hours or days makes it highly infectious. The high proportion of asymptomatic cases and the short doubling time all contribute to the exponential growth of the pandemic. Despite some studies showing an association between an increased weather temperature and reduction in the number of cases, there is no sign that the pandemic will melt away as summer approaches.

Worldwide, unprecedented drastic measures such as movement restrictions, lockdown, airport closures, quarantine and social distancing have been taken to combat the disease. However, despite these vigorous measures, the global number of confirmed cases and deaths is on the rise on a daily basis. Many countries have suffered financially due to months of lockdown and are in a process of unlocking and opening their borders to savage the hard-hit economy. It is important to remember the epidemiological lessons learnt from research as the world embarks on a new normal with the coexistence of SARS-CoV-2. Unless and until a cure or vaccine is established, countries must be constantly on the guard to prevent a second or even third wave of the disease, which is already emerging in some places.

On the other hand, an understanding of the clinical presentation of COVID-19 is important as patients with different presenting complaints and varying degrees of severity require different management strategies. From this review, it can be concluded that the clinical manifestations of SARS-CoV-2 infection are very diverse ranging from no symptoms, to severe disease that requires intensive care and mechanical ventilation, while the most severe cases can lead to death. In the published literature, both pulmonary and extra-pulmonary clinical features have been described, suggesting that the disease can affect nearly any system in the body. Infected individuals who cannot conquer the disease eventually give their lives to COVID-19. However, a majority of people who are infected with SARS-CoV-2 eventually recover from the disease.

Research has also shown that people from all ages can contract SARS-CoV-2 infection. Elderly patients and those with comorbidities are highly susceptible and vulnerable. Earlier studies have claimed that children tend to have milder disease. However, there is evidence that severe and lifethreatening disease can develop in children and that COVID-19 is associated with multisystem inflammatory syndrome and Kawasaki disease in some cases. On the other hand, the clinical presentation in pregnant women is similar 
to that of the general population, whereas there are contradicting findings on immunocompromised individuals with COVID-19. Therefore, it is important that physicians identify the high-risk patients with adverse outcomes and manage them accordingly.

Author Contributions The author contributed solely to the writing and submission of this article. No author who meets the criteria of authorship has been omitted from this submission.

\section{Compliance with Ethical Standards}

Submission Declaration This work has not been published previously and is not under consideration for publication elsewhere.

Conflict of Interest The author declares that she has no conflict of interest.

\section{References}

1. BBC World News. 2020 January 31. Coronavirus declared global health emergency by WHO. Available from https://www.bbc.com/ news/world-51318246 [Accessed 2 June 2020].

2. World Health Organisation. 2020 February 11. WHO DirectorGeneral's remarks at the media briefing on 2019-nCoV on 11 February 2020. Available from https://www.who.int/dg/speeches/ detail/who-director-general-s-remarks-at-the-media-briefing-on2019-ncov-on-11-february-2020 [Accessed 2 June 2020].

3. Business Insider Malaysia. 2020 January 28. Wuhan, China, and at least 15 other cities have been quarantined as China attempts to halt the spread of the coronavirus. That's about 50 million people on lockdown. Available from https://www.businessinsider.my/wuhancoronavirus-officials-quarantine-entire-city-2020-1/? ga $=2$. $223671643.1516976587 .1580894234-855185699$. $1563956440 \& \mathrm{r}=\mathrm{US} \& I R=\mathrm{T}$ [Accessed 2 June 2020].

4. The Guardian, 2020 February 01 . Travel bans plunge China into deepening isolation over coronavirus. Available from https://www. theguardian.com/world/2020/feb/01/coronavirus-travel-bansplunge-china-into-deepening-isolation [Accessed 2 June 2020].

5. World Health Organisation. 2020 March 11. WHO DirectorGeneral's opening remarks at the media briefing on COVID-19 11 March 2020. Available from https://www.who.int/dg/speeches/ detail/who-director-general-s-opening-remarks-at-the-mediabriefing-on-covid-19\%2D\%2D-11-march-2020 [Accessed 2 June 2020].

6. Worldometer 2020. COVID-19 Coronavirus Pandemic. Available from https://www.worldometers.info/coronavirus/ [Accessed 13 September 2020].

7. Li Q, Guan X, Wu P, Wang X, Zhou L, Tong Y, et al. Early transmission dynamics in Wuhan, China, of novel coronavirusinfected pneumonia. N Engl J Med. 2020;382(13):1199-207. https://doi.org/10.1056/NEJMoa2001316.

8. Chen N, Zhou M, Dong X, Qu J, Gong F, Han Y, et al. Epidemiological and clinical characteristics of 99 cases of 2019 novel coronavirus pneumonia in Wuhan, China: a descriptive study [published online ahead of print, 2020 Jan 30]. Lancet. 2020;395(10223):507-13. https://doi.org/10.1016/S01406736(20)30211-7.

9. Lauer SA, Grantz KH, Bi Q, Jones FK, Zheng Q, Meredith HR, et al. The incubation period of coronavirus disease 2019 (COVID-
19) from publicly reported confirmed cases: estimation and application. Ann Intern Med. 2020;172:577-82.

10. Fine PE. The interval between successive cases of an infectious disease. Am J Epidemiol. 2003;158:1039-47.

11. Nishiura H, Linton NM, Akhmetzhanov AR. Serial interval of novel coronavirus (COVID-19) infections. Int J Infect Dis. 2020;93: 284-6. https://doi.org/10.1016/j.ijid.2020.02.060.

12. Liu T, Qi L, Yao M, et al. Serial interval and reproductive number of COVID-19 among 116 infector-infectee pairs - Jingzhou city, Hubei province, China. China CDC Weekly. 2020;2(27):491-5. https://doi.org/10.46234/cedcw2020.118.

13. Aronson JK, Brassey J, Mahtani KR. "When will it be over?": an introduction to viral reproduction numbers, $\mathrm{R}_{0}$ and $\mathrm{R}_{\mathrm{e}}$. The Centre for Evidence-Based Medicine, University of Oxford, 2020. https:// www.cebm.net/covid-19/when-will-it-be-over-an-introduction-toviral-reproduction-numbers-r0-and-re/ Accessed 7 July 2020.

14. Liu Y, Gayle AA, Wilder-Smith A, Rocklöv J. The reproductive number of COVID-19 is higher compared to SARS coronavirus. J Travel Med. 2020;27(2):taaa021. https://doi.org/10.1093/jtm/ taaa021.

15. Caicedo-Ochoa Y, Rebellón-Sánchez DE, Peñaloza-Rallón M, Cortés-Motta HF, Méndez-Fandiño YR. Effective reproductive number estimation for initial stage of COVID-19 pandemic in Latin American countries. Int J Infect Dis. 2020;95:316-8. https:// doi.org/10.1016/j.ijid.2020.04.069.

16. Muniz-Rodriguez K, Chowell G, Cheung CH, et al. Doubling time of the COVID-19 epidemic by Chinese province. Preprint. medRxiv 2020.02.05.20020750. 2020. https://doi.org/10.1101/ 2020.02.05.20020750.

17. Pellis L, Scarabel F, Stage HB, et al. Challenges in control of Covid-19: short doubling time and long delay to effect of interventions. medRxiv. 2020; 2020.04.12.20059972. https://doi.org/10. 1101/2020.04.12.20059972.

18. Huang L, Zhang X, Zhang X, Wei Z, Zhang L, Xu J, et al. Rapid asymptomatic transmission of COVID-19 during the incubation period demonstrating strong infectivity in a cluster of youngsters aged 16-23 years outside Wuhan and characteristics of young patients with COVID-19: a prospective contact-tracing study. J Inf Secur. 2020;80(6):e1-e13. https://doi.org/10.1016/j.jinf.2020.03. 006.

19. Bai Y, Yao L, Wei T, Tian F, Jin DY, Chen L, et al. Presumed asymptomatic carrier transmission of COVID-19. JAMA. 2020;323(14):1406-7. https://doi.org/10.1001/jama.2020.2565.

20. Mizumoto K, Kagaya K, Zarebski A, Chowell G. Estimating the asymptomatic proportion of coronavirus disease 2019 (COVID-19) cases on board the Diamond Princess cruise ship, Yokohama, Japan, 2020. Euro Surveill. 2020;25(10):2000180. https://doi.org/ 10.2807/1560-7917.ES.2020.25.10.2000180.

21. Nishiura H, Kobayashi T, Miyama T, Suzuki A, Jung SM, Hayashi $\mathrm{K}$, et al. Estimation of the asymptomatic ratio of novel coronavirus infections (COVID-19). Int J Infect Dis. 2020;94:154-5. https://doi. org/10.1016/j.ijid.2020.03.020.

22. Fornari F. Vertical transmission of Covid-19 - a systematic review. J Paediatr Perinatol Child Health. 2020;4:007-13.

23. van Doremalen N, Bushmaker T, Morris DH, Holbrook MG, Gamble A, Williamson BN, et al. Aerosol and surface stability of SARS-CoV-2 as compared with SARS-CoV-1. N Engl J Med. 2020;382(16):1564-7. https://doi.org/10.1056/NEJMc2004973.

24. Chin A, Chu J, Perera M, et al. Stability of SARS-CoV-2 in different environmental conditions. medRxiv. 2020. https://doi.org/10. 1101/2020.03.15.20036673.

25. Bhattacharjee S. Statistical investigation of relationship between spread of coronavirus disease (COVID-19) and environmental factors based on study of four mostly affected places of China and five mostly affected places of Italy. arXiv preprint 2020; arXiv: 2003.11277. 
26. Luo W, Majumder MS, Liu D et al. The role of absolute humidity on transmission rates of the COVID-19 outbreak. medRxiv preprint 2020. doi: https://doi.org/10.1101/2020.02.12.20022467.

27. Wu Y, Jing W, Liu J, Ma Q, Yuan J, Wang Y, et al. Effects of temperature and humidity on the daily new cases and new deaths of COVID-19 in 166 countries. Sci Total Environ. 2020;729:139051. https://doi.org/10.1016/j.scitotenv.2020.139051.

28. Hoffmann M, Kleine-Weber H, Krüger N, Müller M, Drosten C, Pöhlmann S. The novel coronavirus 2019 (2019-nCoV) uses the SARS-coronavirus receptor ACE2 and the cellular protease TMPRSS2 for entry into target cells. bioRxiv 2020.01.31.929042; doi: https://doi.org/10.1101/2020.01.31.929042.

29. Mason RJ. Pathogenesis of COVID-19 from a cell biology perspective. Eur Respir J. 2020;55(4):2000607. https://doi.org/10.1183/ 13993003.00607-2020.

30. Wu Z, McGoogan JM. Characteristics of and important lessons from the coronavirus disease 2019 (COVID-19) outbreak in China: summary of a report of 72314 cases from the Chinese Centre for Disease Control and Prevention. JAMA. 2020. https:// doi.org/10.1001/jama.2020.2648.

31. di Mauro G, Cristina S, Concetta R, Francesco R, Annalisa C. SARS-Cov-2 infection: response of human immune system and possible implications for the rapid test and treatment. Int Immunopharmacol. 2020;84:106519. https://doi.org/10.1016/j. intimp.2020.106519.

32. Qin C, Zhou L, Hu Z, Zhang S, Yang S, Tao Y, et al. Dysregulation of immune response in patients with coronavirus 2019 (COVID-19) in Wuhan, China. Clin Infect Dis. 2020;71(15):762-8. https://doi. org/10.1093/cid/ciaa248.

33. Abobaker A, Raba AA, Alzwi A. Extrapulmonary and atypical clinical presentations of COVID-19. J Med Virol. 2020. https:// doi.org/10.1002/jmv.26157.

34. Behzad S, Aghaghazvini L, Radmard AR, Gholamrezanezhad A. Extrapulmonary manifestations of COVID-19: radiologic and clinical overview. Clin Imaging. 2020;66:35-41. https://doi.org/10. 1016/j.clinimag.2020.05.013.

35. Pan L, Mu M, Yang P, Sun Y, Wang R, Yan J, et al. Clinical characteristics of COVID-19 patients with digestive symptoms in Hubei, China: a descriptive, cross-sectional, multicentre study. Am J Gastroenterol. 2020;115(5):766-73. https://doi.org/10.14309/ajg. 0000000000000620

36. Galván Casas C, Català A, Carretero Hernández G, RodríguezJiménez P, Fernández-Nieto D, Rodríguez-Villa Lario A, et al. Classification of the cutaneous manifestations of COVID-19: a rapid prospective nationwide consensus study in Spain with 375 cases. Br J Dermatol. 2020;183(1):71-7. https://doi.org/10.1111/bjd. 19163.

37. Bansal M. Cardiovascular disease and COVID-19. Diabetes Metab Syndr. 2020;14(3):247-50. https://doi.org/10.1016/j.dsx.2020.03. 013.

38. Dong Y, Mo X, Hu Y, Qi X, Jiang F, Jiang Z, et al. Epidemiological characteristics of 2143 paediatric patients with 2019 coronavirus disease in China. Paediatrics. 2020;145(6):e20200702. https://doi. org/10.1542/peds.2020-0702.

39. Greene AG, Saleh M, Roseman E, Sinert R. Toxic shock-like syndrome and COVID-19: a case report of multisystem inflammatory syndrome in children (MIS-C). Am J Emerg Med. 2020;S07356757(20):30492-7. https://doi.org/10.1016/j.ajem.2020.05.117.

40. Toubiana J, Poirault C, Corsia A, Bajolle F, Fourgeaud J, Angoulvant F, et al. Kawasaki-like multisystem inflammatory syndrome in children during the covid-19 pandemic in Paris, France: prospective observational study. BMJ. 2020;369:m2094.

41. Cao D, Yin H, Chen J, Tang F, Peng M, Li R, et al. Clinical analysis of ten pregnant women with COVID-19 in Wuhan, China: a retrospective study. Int J Infect Dis. 2020;95:294-300. https://doi.org/ 10.1016/j.ijid.2020.04.047.

42. Matar R, Alrahmani L, Monzer N, et al. Clinical presentation and outcomes of pregnant women with COVID-19: a systematic review and meta-analysis. Clin Infect Dis. 2020:ciaa828. https://doi.org/10. 1093/cid/ciaa828.

43. Gervasoni C, Meraviglia P, Riva A, Giacomelli A, Oreni L, Minisci $\mathrm{D}$, et al. Clinical features and outcomes of HIV patients with coronavirus disease 2019. Clin Infect Dis:ciaa579. https://doi.org/10. 1093/cid/ciaa579.

44. Davis M. HIV and risk of COVID-19 death: a population cohort study from the Western Cape Province, South Africa. medRxiv. 2020. https://doi.org/10.1101/2020.07.02.20145185.

45. Lee LYW, Cazier JB, Starkey T, et al. COVID-19 mortality in patients with cancer on chemotherapy or other anticancer treatments: a prospective cohort study. Lancet. 2020;395(10241): 1919-26. https://doi.org/10.1016/S0140-6736(20)31173-9.

46. Yang K, Sheng Y, Huang C, Jin Y, Xiong N, Jiang K, et al. Clinical characteristics, outcomes, and risk factors for mortality in patients with cancer and COVID-19 in Hubei, China: a multicentre, retrospective, cohort study. Lancet Oncol. 2020;21(7):904-13. https:// doi.org/10.1016/S1470-2045(20)30310-7.

47. Onder G, Rezza G, Brusaferro S. Case-fatality rate and characteristics of patients dying in relation to COVID-19 in Italy. JAMA. 2020;323(18):1775-6. https://doi.org/10.1001/jama.2020.4683.

48. Richardson S, Hirsch JS, Narasimhan M, Crawford JM, McGinn T, Davidson KW, et al. Presenting characteristics, comorbidities, and outcomes among 5700 patients hospitalized with COVID-19 in the New York City area. JAMA. 2020;323(20):2052-9. https://doi.org/ 10.1001/jama.2020.6775.

49. Guan WJ, Liang WH, Zhao Y, et al. Comorbidity and its impact on 1590 patients with COVID-19 in China: a nationwide analysis. Eur Respir J. 2020;55(5):2000547. Published 2020 May 14. https://doi. org/10.1183/13993003.00547-2020.

Publisher's Note Springer Nature remains neutral with regard to jurisdictional claims in published maps and institutional affiliations. 